\title{
Velocity Averaging Lemmas in Hyperbolic Sobolev Spaces for the Kinetic Transport Equation with Velocity Field on the Sphere
}

Nikolaos Bournaveas and Hua Wang

\begin{abstract}
We show how the methods of [6-8] can be used to prove velocity averaging lemmas in hyperbolic Sobolev spaces for the kinetic transport equation $\partial_{t} f+\frac{v}{|v|} \cdot \nabla_{x} f=g_{0}+\nabla_{v} \cdot g_{1}$. Here $v$ is allowed to vary in the whole space $\mathbb{R}^{d}$ and the velocity field $a(v)=\frac{v}{|v|}$ lies on the unit sphere. We work in dimensions $d \geq 3$ and, in contrast with [6,8], we allow right-hand sides with velocity derivatives in any direction and not necessarily tangential to the sphere.
\end{abstract}

Mathematics Subject Classification (2000). 82C70, 35B45, 35F10, 35Q75.

Keywords. Velocity averaging, hyperbolic Sobolev spaces, chemotaxis.

\section{Introduction}

In this paper we show how the methods of [6-8] can be used to prove velocity averaging lemmas in hyperbolic Sobolev spaces $H^{s, \delta}$ for the kinetic transport equation

$$
\partial_{t} f+\frac{v}{|v|} \cdot \nabla_{x} f=g_{0}+\nabla_{v} \cdot g_{1} .
$$

Velocity averaging lemmas were first proved in $[17,18]$ and later developed further in several papers $[3,4,11,12,14-16,19,20,23-27,29]$. We refer the reader to $[3,28]$ for a review and an extensive bibliography. In the simplest setting the velocity average over the unit ball $\rho(t, x)=\int_{|v| \leq 1} f(t, x, v) d v$ of a solution $f$ of

$$
\partial_{t} f+v \cdot \nabla_{x} f=g,
$$

gains half a derivative in $L^{2}$. More precisely, if $f, g \in L^{2}\left(\mathbb{R}_{t} \times \mathbb{R}_{x}^{d} \times \mathbb{R}_{v}^{d}\right)$ satisfy (1.2), then

$$
\|\rho\|_{H^{1 / 2}\left(\mathbb{R}_{t} \times \mathbb{R}_{x}^{d}\right)} \lesssim\|f\|_{L^{2}\left(\mathbb{R}_{t} \times \mathbb{R}_{x}^{d} \times \mathbb{R}_{v}^{d}\right)}+\|g\|_{L^{2}\left(\mathbb{R}_{t} \times \mathbb{R}_{x}^{d} \times \mathbb{R}_{v}^{d}\right)} .
$$


Averages over spheres come up in many applications where the velocity of particles can be assumed to be constant, for example in radiative transfer and chemotaxis (see Section 3). These averages were studied in [6-8]. When the right hand side doesn't contain velocity derivatives it was shown that

$$
\|\rho\|_{H^{s, \delta}\left(\mathbb{R}^{1+d}\right)} \leq C\|f\|_{L^{2}\left(\mathbb{R} \times \mathbb{R}^{d} \times S^{d-1}\right)}+C\|g\|_{L^{2}\left(\mathbb{R} \times \mathbb{R}^{d} \times S^{d-1}\right)},
$$

provided that

$$
s+\delta \leq 1 / 2, \quad s \leq \min \left\{\frac{d-1}{4}, 1\right\}, \quad d \geq 3, \quad d \neq 7 .
$$

where $f, g$ solve (1.2), $\rho$ is given by $\rho(t, x)=\int_{|v|=1} f(t, x, v) d \sigma_{v}$, and the hyperbolic Sobolev norm is defined by

$$
\|F\|_{H^{s, \delta}\left(\mathbb{R}^{1+d}\right)}=\left\|w_{+}(\tau, \xi)^{s} w_{-}(\tau, \xi)^{\delta} \widehat{F}(\tau, \xi)\right\|_{L^{2}\left(\mathbb{R}_{\tau} \times \mathbb{R}_{\xi}^{d}\right)}
$$

where $w_{ \pm}(\tau, \xi)=1+\| \tau| \pm| \xi||$. The advantage is that we can take $s$ larger than the classical $1 / 2$ provided that we compensate by using a negative $\delta$. Moreover, in regions where ||$\tau|-| \xi||$ is small (near the cone $|\tau|=|\xi|$ ) we have $w_{+}^{s} w_{-}^{\delta} \simeq w_{+}^{s}$ and we gain $s \geq 1 / 2$ derivatives. In all regions we have $w_{+}(\tau, \xi)^{s} w_{-}(\tau, \xi)^{\delta} \geq$ $w_{+}(\tau, \xi)^{1 / 2}$, therefore estimate (1.3) implies the classical estimate. When $d=7$ we have the same estimate but with a logarithmic loss:

$$
\|\rho\|_{H_{\log }^{s, \delta}\left(R^{1+d}\right)} \leq C\left(\|f\|_{L^{2}\left(R \times R^{d} \times S^{d-1}\right)}+\|g\|_{L^{2}\left(R \times R^{d} \times S^{d-1}\right)}\right), \quad d=7,
$$

where

$$
\begin{aligned}
& \|F\|_{H_{\log }^{s, \delta}\left(\mathbb{R}^{1+d}\right)} \\
& \quad=\left\|w_{+}(\tau, \xi)^{s} w_{-}(\tau, \xi)^{\delta}\left(1+\log \frac{w_{+}(\tau, \xi)}{w_{-}(\tau, \xi)}\right)^{-1 / 2} \widehat{F}(\tau, \xi)\right\|_{L^{2}\left(\mathbb{R}_{\tau} \times \mathbb{R}_{\xi}^{d}\right)} .
\end{aligned}
$$

Consider now the case of a right hand side which contains velocity derivatives. In this case a crucial step in the proof of averaging lemmas is an integration by parts in velocity space which removes the $v$-derivative from $g$. In the case of spheres this can be done only if the velocity derivatives are tangential to the sphere, i.e. if $f$ and $g$ solve

$$
\partial_{t} f+v \cdot \nabla_{x} f=\Omega_{v}^{i, j} g
$$

where $\Omega_{v}^{i, j}=v_{i} \frac{\partial}{\partial v_{j}}-v_{j} \frac{\partial}{\partial v_{i}}$. We refer the reader to [6-8] for a detailed study.

In this paper we study velocity averages of the form

$$
\rho(t, x)=\int_{\mathbb{R}^{d}} f(t, x, v) \phi(v) d v,
$$

where $\phi(v)$ is a smooth cut-off function and $f$ is a solution of equation (1.1). We allow general (i.e. not necessarily tangential) velocity derivatives in the right hand side. To deal with this case we shall decompose the velocity derivative into radial and tangential components and use the methods of [6] to handle the tangential 
part. The radial part can be handled by an integration by parts in the radial variable. More precisely we prove the following two theorems:

Theorem 1.1. Let $d \geq 2$ and let $f, g: \mathbb{R}_{t} \times \mathbb{R}_{x}^{d} \times \mathbb{R}_{v}^{d} \rightarrow \mathbb{R}$ solve

$$
\partial_{t} f+\frac{v}{|v|} \cdot \nabla_{x} f=g .
$$

Let $\phi(v) \in L^{\infty}$ and suppose that $\phi$ has compact support contained in some ball $B$ centered at the origin. Define

$$
\rho(t, x)=\int_{\mathbb{R}^{d}} f(t, x, v) \phi(v) d v,
$$

and $s=\min \left\{\frac{d-1}{4}, 1\right\}, \delta=\frac{1}{2}-s$. Then

$$
\begin{array}{ll}
\|\rho\|_{H^{s, \delta}\left(\mathbb{R} \times \mathbb{R}^{d}\right)} \lesssim\left[\|f\|_{L^{2}\left(\mathbb{R} \times \mathbb{R}^{d} \times B\right)}+\|g\|_{L^{2}\left(\mathbb{R} \times \mathbb{R}^{d} \times B\right)}\right], & d \neq 5, \\
\|\rho\|_{H_{\text {log }}^{s, \delta}\left(\mathbb{R} \times \mathbb{R}^{d}\right)} \lesssim\left[\|f\|_{L^{2}\left(\mathbb{R} \times \mathbb{R}^{d} \times B\right)}+\|g\|_{L^{2}\left(\mathbb{R} \times \mathbb{R}^{d} \times B\right)}\right], & d=5 .
\end{array}
$$

Notice that in $d=2$ dimensions we get an estimate in the hyperbolic Sobolev space $H^{1 / 4,1 / 4}$ as in Theorem 2 of [6]. In $d \geq 3$ dimensions we have $s \geq 1 / 2$ and $\delta \leq 0$ with sum $s+\delta=1 / 2$, hence $w_{+}^{s} w_{-}^{\delta}=w_{+}^{1 / 2}\left(w_{+} / w_{-}\right)^{s-1 / 2} \geq w_{+}^{1 / 2}$. Therefore our estimate implies the classical gain of $1 / 2$ derivatives.

When the right hand side contains velocity derivatives we prove the following

Theorem 1.2. Let $d \geq 3$ and let $f, g: \mathbb{R}_{t} \times \mathbb{R}_{x}^{d} \times \mathbb{R}_{v}^{d} \rightarrow \mathbb{R}$ solve

$$
\partial_{t} f+\frac{v}{|v|} \cdot \nabla_{x} f=\nabla_{v} \cdot g \text {. }
$$

Let $\phi(v) \in W^{1, \infty}\left(\mathbb{R}^{d}\right)$ with $\operatorname{supp} \phi \subseteq B$ where $B$ is a ball centered at the origin. Define the velocity average

$$
\rho(t, x)=\int_{\mathbb{R}^{d}} f(t, x, v) \phi(v) d v .
$$

Let

$$
\begin{aligned}
& (s, \delta)=\left(\frac{d-1}{6},-\frac{2 d-5}{12}\right), \quad \text { if } \quad d \in\{3,4,5\} \\
& (s, \delta)=\left(\frac{4}{5},-\frac{11}{20}\right), \quad \text { if } \quad d=6 \\
& (s, \delta)=\left(1,-\frac{3}{4}\right), \quad \text { if } \quad d \geq 7 .
\end{aligned}
$$

Then

$$
\begin{aligned}
& \|\rho\|_{H^{s, \delta}\left(\mathbb{R}^{1+d}\right)} \lesssim\|f\|_{L^{2}\left(\mathbb{R} \times \mathbb{R}^{d} \times B\right)}+\|g\|_{L^{2}\left(\mathbb{R} \times \mathbb{R}^{d} \times B\right)}, \quad \text { if } \quad d \neq 5,7, \\
& \|\rho\|_{H_{\log }^{s, \delta}\left(\mathbb{R}^{1+d}\right)} \lesssim\|f\|_{L^{2}\left(\mathbb{R} \times \mathbb{R}^{d} \times B\right)}+\|g\|_{L^{2}\left(\mathbb{R} \times \mathbb{R}^{d} \times B\right)}, \quad \text { if } \quad d=5,7 \text {. }
\end{aligned}
$$


Notice that in all cases $s+\delta=\frac{1}{4}$ with $s>\frac{1}{4}$ and $\delta<0$. Moreover, $w_{+}^{s} w_{-}^{\delta}=$ $w_{+}^{\frac{1}{4}-\delta} w_{-}^{\delta}=w_{+}^{\frac{1}{4}}\left(\frac{w_{+}}{w_{-}}\right)^{-\delta} \geq w_{+}^{\frac{1}{4}}$, therefore our result implies the classical gain of $1 / 4$ derivatives.

The decomposition of the velocity derivative into tangential and radial parts (we also add $\lambda f$ to both sides of the equation) creates three different terms (see (2.21)) with different scaling properties and results in the more complicated conditions on the dimension in Theorem 1.2.

We shall prove Theorems 1.1 and 1.2 in Section 2 and we shall discuss applications in Biology and Physics in Section 3.

\section{Proofs of the velocity averaging estimates}

We recall the following estimates from [7].

Lemma 2.1. Let $m>-1, l>1 / 2$ and define

$$
J_{l}^{m}(\tau, \xi)=\int_{0}^{\pi} \frac{(\sin \theta)^{m}}{\left[1+(\tau+|\xi| \cos \theta)^{2}\right]^{l}} d \theta, \quad \tau \in \mathbb{R}, \quad \xi \in \mathbb{R}^{d} .
$$

Let $\alpha=\min \left\{\frac{m+1-4 l}{2}, 0\right\}$. Then the integrals $J_{l}^{m}(\tau, \xi)$ satisfy the following pointwise estimates:

$$
\begin{aligned}
& J_{l}^{m}(\tau, \xi) \lesssim \frac{w_{-}(\tau, \xi)^{2 l-1+\alpha}}{w_{+}(\tau, \xi)^{2 l+\alpha}}, \quad \text { if } \quad m+1 \neq 4 l \\
& J_{l}^{m}(\tau, \xi) \lesssim \frac{w_{-}(\tau, \xi)^{2 l-1}}{w_{+}(\tau, \xi)^{2 l}}\left(1+\log \frac{w_{+}(\tau, \xi)}{w_{-}(\tau, \xi)}\right), \quad \text { if } \quad m+1=4 l .
\end{aligned}
$$

Proof. See Proposition 3.1 in [7].

Proof of Theorem 1.1. Define

$$
w(\tau, \xi)=\left\{\begin{array}{lll}
w_{+}(\tau, \xi)^{s} w_{-}(\tau, \xi)^{\delta}, & \text { if } \quad d \neq 5 \\
\frac{w_{+}(\tau, \xi)^{s} w_{-}(\tau, \xi)^{\delta}}{\left[1+\log \frac{w_{+}(\tau, \xi)}{w_{-}(\tau, \xi)}\right]^{1 / 2}}, & \text { if } \quad d=5 .
\end{array} .\right.
$$

where $s=\min \left\{\frac{d-1}{4}, 1\right\}$ and $\delta=\frac{1}{2}-s$. For each $r>0$ define $f_{r}, g_{r}: \mathbb{R} \times \mathbb{R}^{d} \times \mathbb{S}^{d-1} \rightarrow$ $\mathbb{R}$ by $f_{r}(t, x, \omega)=f(t, x, r \omega)$ and $g_{r}(t, x, \omega)=g(t, x, r \omega)$. Then

$$
\partial_{t} f_{r}+\omega \cdot \nabla_{x} f_{r}=g_{r} .
$$

Take the Fourier transform with respect to $t$ and $x$ and add $\widehat{f}_{r}$ to both sides to get

$$
\widehat{f}_{r}(\tau, \xi, \omega)=\frac{\widehat{h_{r}}(\tau, \xi, \omega)}{1+i(\tau+\omega \cdot \xi)}
$$


where $h=f+g$. Therefore,

$$
\begin{aligned}
\widehat{\rho}(\tau, \xi) & =\int_{0}^{\infty} \int_{\mathbb{S}^{d-1}} r^{d-1} \widehat{f}_{r}(\tau, \xi, \omega) \phi(r \omega) d \omega d r \\
& =\int_{0}^{\infty} \int_{\mathbb{S}^{d-1}} r^{d-1} \frac{\widehat{h_{r}}(\tau, \xi, \omega)}{1+i(\tau+\omega \cdot \xi)} \phi(r \omega) d \omega d r .
\end{aligned}
$$

Use the Cauchy-Schwarz inequality on $\mathbb{S}^{d-1}$ to get

$$
\begin{aligned}
|\widehat{\rho}(\tau, \xi)| \leq & \int_{0}^{\infty} r^{d-1}\left(\int_{\mathbb{S}^{d-1}} \frac{1}{1+(\tau+\omega \cdot \xi)^{2}} d \omega\right)^{1 / 2} \\
& \cdot\left(\int_{\mathbb{S}^{d-1}}\left|\widehat{h_{r}}(\tau, \xi, \omega) \phi(r \omega)\right|^{2} d \omega\right)^{1 / 2} d r .
\end{aligned}
$$

We have

$$
\begin{aligned}
\int_{\mathbb{S}^{d-1}} \frac{1}{1+(\tau+\omega \cdot \xi)^{2}} d \omega & =c(d) \int_{0}^{\pi} \frac{(\sin \theta)^{d-2}}{1+(\tau+|\xi| \cos \theta)^{2}} d \theta \\
& =c(d) J_{l}^{m}(\tau, \xi)
\end{aligned}
$$

where $m=d-2$ and $l=1$. For $d \neq 5$ Lemma 2.1 gives:

$$
\begin{aligned}
\left(\int_{\mathbb{S}^{d-1}} \frac{1}{1+(\tau+\omega \cdot \xi)^{2}} d \omega\right)^{1 / 2} & \lesssim\left(\frac{w_{-}(\tau, \xi)^{2 s-1}}{w_{+}(\tau, \xi)^{2 s}}\right)^{1 / 2} \\
& =\frac{1}{w_{+}(\tau, \xi)^{s} w_{-}(\tau, \xi)^{\delta}}
\end{aligned}
$$

For $d=5$ we get

$$
\begin{aligned}
\left(\int_{\mathbb{S}^{d-1}} \frac{1}{1+(\tau+\omega \cdot \xi)^{2}}\right. & d \omega)^{1 / 2} \\
& \lesssim \frac{1}{w_{+}(\tau, \xi)^{s} w_{-}(\tau, \xi)^{\frac{1}{2}-s}}\left[1+\log \frac{w_{+}(\tau, \xi)}{w_{-}(\tau, \xi)}\right]^{1 / 2}
\end{aligned}
$$

This proves that in all cases

$$
\left(\int_{\mathbb{S}^{d-1}} \frac{1}{1+(\tau+\omega \cdot \xi)^{2}} d \omega\right)^{1 / 2} \lesssim \frac{1}{w(\tau, \xi)}
$$

with $w(\tau, \xi)$ as in (2.4). Therefore

$$
w(\tau, \xi)|\widehat{\rho}(\tau, \xi)| \lesssim \int_{0}^{\infty} r^{d-1}\left(\int_{\mathbb{S}^{d-1}}\left|\widehat{h_{r}}(\tau, \xi, \omega) \phi(r \omega)\right|^{2} d \omega\right)^{1 / 2} d r
$$


Let $R>0$ be such that $\operatorname{supp} \phi \subseteq B(0, R)$. Then

$$
\begin{aligned}
w(\tau, \xi) \mid & \hat{\rho}(\tau, \xi) \mid \\
& \lesssim \int_{0}^{R} r^{\frac{d-1}{2}} r^{\frac{d-1}{2}}\left(\int_{\mathbb{S}^{d-1}}\left|\widehat{h_{r}}(\tau, \xi, \omega) \phi(r \omega)\right|^{2} d \omega\right)^{1 / 2} d r \\
& \lesssim\left(\int_{0}^{R} r^{d-1} d r\right)^{1 / 2}\left(\int_{0}^{R} \int_{\mathbb{S}^{d-1}} r^{d-1}|\widehat{h}(\tau, \xi, r \omega) \phi(r \omega)|^{2} d \omega\right)^{1 / 2} \\
& \lesssim\|\widehat{h}(\tau, \xi, v) \phi(v)\|_{L_{v}^{2}(B(0, R))} \\
& \lesssim\|\widehat{h}(\tau, \xi, v)\|_{L_{v}^{2}(B(0, R))} .
\end{aligned}
$$

This implies the desired estimate (1.9).

Proof of Theorem 1.2. The idea is to decompose the velocity derivative into radial and angular parts and deal with the angular parts by the method introduced in [6]. It suffices to show:

$$
w(\tau, \xi)|\widehat{\rho}(\tau, \xi)| \lesssim\left[\|\widehat{f}(\tau, \xi, v)\|_{L_{v}^{2}(B)}+\|\widehat{g}(\tau, \xi, v)\|_{L_{v}^{2}(B)}\right]
$$

where

$$
w(\tau, \xi)= \begin{cases}w_{+}(\tau, \xi)^{s} w_{-}(\tau, \xi)^{\delta}, & d \geq 3, \quad d \neq 5,7 \\ w_{+}(\tau, \xi)^{s} w_{-}(\tau, \xi)^{\delta}\left(1+\log \frac{w_{+}(\tau, \xi)}{w_{-}(\tau, \xi)}\right)^{-1 / 2}, & d=5,7,\end{cases}
$$

and $s, \delta$ are given by (1.12).

Write $v=r \omega$, where $r=|v|$ and $\omega=\frac{v}{|v|} \in \mathbb{S}^{d-1}$. Then

$$
\partial_{t} f+\omega \cdot \nabla_{x} f=\sum_{j} \frac{\partial g^{j}}{\partial v_{j}}=\sum_{j} \omega_{j} \frac{\partial g^{j}}{\partial r}+\frac{1}{r} \sum_{i, j} \omega_{i} \Omega_{\omega}^{i, j} g^{j}
$$

where $\Omega_{\omega}^{i, j}=\omega_{i} \frac{\partial}{\partial \omega_{j}}-\omega_{j} \frac{\partial}{\partial \omega_{i}}$. Take the Fourier transform of the equation and add $\lambda(\tau, \xi) \widehat{f}(\tau, \xi, r \omega)$ to both sides, where $\lambda(\tau, \xi) \geq 1$ will be determined later, to get

$$
\begin{aligned}
\widehat{f}(\tau, \xi, \omega)= & \sum_{j} \frac{\omega_{j} \frac{\partial}{\partial r}\left\{\widehat{g^{j}}(\tau, \xi, r \omega)\right\}}{\lambda+i(\tau+\omega \cdot \xi)}+\frac{1}{r} \sum_{i, j} \frac{\omega_{i} \Omega_{\omega}^{i, j} \widehat{g^{j}}(\tau, \xi, r \omega)}{\lambda+i(\tau+\omega \cdot \xi)} \\
& +\frac{\lambda \widehat{f}(\tau, \xi, \omega)}{\lambda+i(\tau+\omega \cdot \xi)},
\end{aligned}
$$


therefore

$$
\begin{aligned}
\widehat{\rho}(\tau, \xi)= & \int_{0}^{\infty} \int_{\mathbb{S}^{d-1}} \widehat{f}(\tau, \xi, r \omega) \phi(r \omega) r^{d-1} d \omega d r \\
= & \sum_{j} \int_{0}^{\infty} \int_{\mathbb{S}^{d-1}} \frac{\omega_{j} \frac{\partial}{\partial r}\left\{\widehat{g^{j}}(\tau, \xi, r \omega)\right\}}{\lambda+i(\tau+\omega \cdot \xi)} \phi(r \omega) r^{d-1} d \omega d r \\
& +\sum_{i, j} \int_{0}^{\infty} \int_{\mathbb{S}^{d-1}} \frac{\omega_{i} \Omega_{\omega}^{i, j} \widehat{g^{j}}(\tau, \xi, r \omega)}{\lambda+i(\tau+\omega \cdot \xi)} \phi(r \omega) r^{d-2} d \omega d r \\
& +\int_{0}^{\infty} \int_{\mathbb{S}^{d-1}} \frac{\lambda \widehat{f}(\tau, \xi, r \omega)}{\lambda+i(\tau+\omega \cdot \xi)} \phi(r \omega) r^{d-1} d \omega d r \\
= & : \sum_{j} A^{j}(\tau, \xi)+\sum_{i, j} B^{i j}(\tau, \xi)+C(\tau, \xi) .
\end{aligned}
$$

We start with $A^{j}$. Integrating by parts in the radial variable we have

$$
\begin{aligned}
A^{j}(\tau, \xi)= & -\int_{0}^{\infty} \int_{\mathbb{S}^{d-1}} \frac{\omega_{j} \widehat{g^{j}}(\tau, \xi, r \omega)}{\lambda+i(\tau+\omega \cdot \xi)} \frac{\partial}{\partial r}\left[\phi(r \omega) r^{d-1}\right] d \omega d r \\
= & -\int_{0}^{\infty} \int_{\mathbb{S}^{d-1}} \frac{\omega_{j} \widehat{g^{j}}(\tau, \xi, r \omega)}{\lambda+i(\tau+\omega \cdot \xi)} \nabla \phi(r \omega) \cdot \omega r^{d-1} d \omega d r \\
& -(d-1) \int_{0}^{\infty} \int_{\mathbb{S}^{d-1}} \frac{\omega_{j} \widehat{g^{j}}(\tau, \xi, r \omega)}{\lambda+i(\tau+\omega \cdot \xi)} \phi(r \omega) r^{d-2} d \omega d r,
\end{aligned}
$$

therefore

$$
\begin{aligned}
& \left|A^{j}(\tau, \xi)\right| \\
& \lesssim \int_{0}^{\infty}\left(\int_{\mathbb{S}^{d-1}} \frac{d \omega}{\lambda^{2}+(\tau+\omega \cdot \xi)^{2}}\right)^{1 / 2} . \\
& \cdot\left(\int_{\mathbb{S}^{d-1}}\left|\omega_{j} \widehat{g^{j}}(\tau, \xi, r \omega) \nabla \phi(r \omega) \cdot \omega\right|^{2} d \omega\right)^{1 / 2} r^{d-1} d r \\
& +\int_{0}^{\infty}\left(\int_{\mathbb{S}^{d-1}} \frac{d \omega}{\lambda^{2}+(\tau+\omega \cdot \xi)^{2}}\right)^{1 / 2}\left(\int_{\mathbb{S}^{d-1}}\left|\omega_{j} \widehat{g^{j}}(\tau, \xi, r \omega) \phi(r \omega)\right|^{2} d \omega\right)^{1 / 2} r^{d-2} d r .
\end{aligned}
$$

Since $\lambda \geq 1$ estimate (2.10) gives

$$
\begin{aligned}
\left(\int_{\mathbb{S}^{d-1}} \frac{1}{\lambda^{2}+(\tau+\omega \cdot \xi)^{2}} d \omega\right)^{1 / 2} & \lesssim\left(\int_{\mathbb{S}^{d-1}} \frac{1}{1+(\tau+\omega \cdot \xi)^{2}} d \omega\right)^{1 / 2} \\
& \lesssim \frac{1}{w_{+}(\tau, \xi)^{\frac{1}{2}}}
\end{aligned}
$$


138

N. Bournaveas and H. Wang

NoDE

therefore

$(2.24)$

$$
\begin{aligned}
& w_{+}(\tau, \xi)^{1 / 2}\left|A^{j}(\tau, \xi)\right| \\
& \lesssim\left(\int_{0}^{\infty} \int_{\mathbb{S}^{d-1}}\left|\omega_{j} \widehat{g^{j}}(\tau, \xi, r \omega) \nabla \phi(r \omega) \cdot \omega\right|^{2} r^{d-1} d \omega d r\right)^{1 / 2}\left(\int_{0}^{R} r^{d-1} d r\right)^{1 / 2} \\
& \quad+\left(\int_{0}^{\infty} \int_{\mathbb{S}^{d-1}}\left|\omega_{j} \widehat{g^{j}}(\tau, \xi, r \omega) \phi(r \omega)\right|^{2} r^{d-1} d \omega d r\right)^{1 / 2}\left(\int_{0}^{R} r^{d-3} d r\right)^{1 / 2} \\
& \lesssim \frac{1}{w_{+}(\tau, \xi)^{1 / 2}}\left\|\widehat{g^{j}}(\tau, \xi, v)\right\|_{L_{v}^{2}(B)} .
\end{aligned}
$$

For $B^{i j}(\tau, \xi)$ we integrate by parts on the sphere to get

$$
\begin{aligned}
B^{i j}(\tau, \xi)= & -\int_{0}^{\infty} \int_{\mathbb{S}^{d-1}}\left[\Omega_{\omega}^{i, j} \frac{1}{\lambda+i(\tau+\omega \cdot \xi)}\right] \omega_{i} \widehat{g^{j}}(\tau, \xi, r \omega) \phi(r \omega) r^{d-2} d \omega d r \\
& -\int_{0}^{\infty} \int_{\mathbb{S}^{d-1}} \frac{1}{\lambda+i(\tau+\omega \cdot \xi)} \Omega_{\omega}^{i, j}\left[\omega_{i} \phi(r \omega)\right] \widehat{g^{j}}(\tau, \xi, r \omega) r^{d-2} d \omega d r \\
= & i \int_{0}^{\infty} \int_{\mathbb{S}^{d-1}} \frac{\omega_{i} \xi_{j}-\omega_{j} \xi_{i}}{(\lambda+i(\tau+\omega \cdot \xi))^{2}} \omega_{i} \widehat{g^{j}}(\tau, \xi, r \omega) \phi(r \omega) r^{d-2} d \omega d r \\
& -\int_{0}^{\infty} \int_{\mathbb{S}^{d-1}} \frac{1}{\lambda+i(\tau+\omega \cdot \xi)} \Omega_{\omega}^{i, j}\left[\omega_{i} \phi(r \omega)\right] \widehat{g^{j}}(\tau, \xi, r \omega) r^{d-2} d \omega d r .
\end{aligned}
$$

Therefore

$$
\begin{aligned}
& \left|B^{i j}(\tau, \xi)\right| \\
\leq & \int_{0}^{\infty}\left(\int_{\mathbb{S}^{d-1}} \frac{\left(\omega_{i} \xi_{j}-\omega_{j} \xi_{i}\right)^{2} d \omega}{\left(\lambda^{2}+(\tau+\omega \cdot \xi)^{2}\right)^{2}}\right)^{1 / 2}\left(\int_{\mathbb{S}^{d-1}}\left|\omega_{i} \widehat{g^{j}}(\tau, \xi, r \omega) \phi(r \omega)\right|^{2} d \omega\right)^{1 / 2} r^{d-2} d r \\
& +\int_{0}^{\infty}\left(\int_{\mathbb{S}^{d-1}} \frac{d \omega}{\lambda^{2}+(\tau+\omega \cdot \xi)^{2}}\right)^{1 / 2} . \\
& \cdot \quad\left(\int_{\mathbb{S}^{d-1}}\left|\Omega_{\omega}^{i, j}\left[\omega_{i} \phi(r \omega)\right]\right|^{2}\left|\widehat{g^{j}}(\tau, \xi, r \omega)\right|^{2} d \omega\right)^{1 / 2} r^{d-2} d r \\
= & : X^{i j}(\tau, \xi)+Y^{i j}(\tau, \xi) .
\end{aligned}
$$

For $Y^{i j}(\tau, \xi)$ we use $(2.23)$ to get

$$
\begin{aligned}
Y^{i j}(\tau, \xi) & \lesssim \frac{1}{w_{+}(\tau, \xi)^{1 / 2}} \int_{0}^{\infty}\left(\int_{\mathbb{S}^{d-1}}\left|\widehat{g^{j}}(\tau, \xi, r \omega)\right|^{2} d \omega\right)^{1 / 2} r^{d-2} d r \\
& \lesssim \frac{1}{w_{+}(\tau, \xi)^{1 / 2}}\left(\int_{0}^{R} \int_{\mathbb{S}^{d-1}}\left|\widehat{g^{j}}(\tau, \xi, r \omega)\right|^{2} r^{d-1} d \omega d r\right)^{1 / 2}\left(\int_{0}^{R} r^{d-3} d r\right)^{1 / 2} \\
& \lesssim \frac{1}{w_{+}(\tau, \xi)^{1 / 2}}\left\|\widehat{g^{j}}(\tau, \xi, v)\right\|_{L_{v}^{2}(B)} .
\end{aligned}
$$


For $X^{i j}(\tau, \xi)$ we have

$$
\begin{aligned}
X^{i j}(\tau, \xi) & \leq K(\tau, \xi) \int_{0}^{\infty}\left(\int_{\mathbb{S}^{d-1}}\left|\omega_{i} \widehat{g^{j}}(\tau, \xi, r \omega) \phi(r \omega)\right|^{2} d \omega\right)^{1 / 2} r^{d-2} d r \\
& \lesssim K(\tau, \xi)\left(\int_{0}^{R} \int_{\mathbb{S}^{d-1}}\left|\widehat{g^{j}}(\tau, \xi, r \omega)\right|^{2} r^{d-1} d \omega d r\right)^{1 / 2}\left(\int_{0}^{R} r^{d-3} d r\right)^{1 / 2} \\
& \lesssim K(\tau, \xi)\left\|\widehat{g^{j}}(\tau, \xi, v)\right\|_{L_{v}^{2}(B)},
\end{aligned}
$$

where

$$
K(\tau, \xi)=\left(\int_{\mathbb{S}^{d-1}} \frac{\left(\omega_{i} \xi_{j}-\omega_{j} \xi_{i}\right)^{2}}{\left(\lambda^{2}+(\tau+\omega \cdot \xi)^{2}\right)^{2}} d \omega\right)^{1 / 2}
$$

For $C(\tau, \xi)$ we have:

$$
\begin{aligned}
C(\tau, \xi) & \leq L(\tau, \xi) \int_{0}^{\infty}\left(\int_{\mathbb{S}^{d-1}}|\widehat{f}(\tau, \xi, r \omega) \phi(r \omega)|^{2} d \omega\right)^{1 / 2} r^{d-1} d r \\
& \lesssim L(\tau, \xi) \int_{0}^{\infty}\left(\int_{0}^{R} \int_{\mathbb{S}^{d-1}}|\widehat{f}(\tau, \xi, r \omega)|^{2} r^{d-1} d \omega d r\right)^{1 / 2}\left(\int_{0}^{R} r^{d-1} d r\right)^{1 / 2} \\
& \lesssim L(\tau, \xi)\|\widehat{f}(\tau, \xi, v)\|_{L_{v}^{2}(B)}
\end{aligned}
$$

where

$$
L(\tau, \xi)=\lambda\left(\int_{\mathbb{S}^{d-1}} \frac{1}{\lambda^{2}+(\tau+\omega \cdot \xi)^{2}} d \omega\right)^{1 / 2} .
$$

It was shown in [8] that there exists a choice of $\lambda=\lambda(\tau, \xi) \geq 1$ such that

$$
K(\tau, \xi) \lesssim w(\tau, \xi), \quad L(\tau, \xi) \lesssim w(\tau, \xi)
$$

This completes the proof.

\section{Motivation and Perspectives}

Velocity fields on the sphere arise naturally in mathematical Biology in the context of chemotaxis. This is the directed motion of cells towards higher concentrations of a chemoattractant. At the mesoscopic level chemotaxis can be modelled by the Othmner-Dunbar-Alt kinetic model [9, 10,22]:

$$
\begin{aligned}
\partial_{t} f+v \cdot \nabla_{x} f= & \int_{V} T[S]\left(t, x, v, v^{\prime}\right) f\left(t, x, v^{\prime}\right) d v^{\prime} \\
& -\int_{V} T[S]\left(t, x, v^{\prime}, v\right) f(t, x, v) d v^{\prime}, \\
-\Delta S= & \alpha \rho-\beta S,
\end{aligned}
$$


where $f$ is the density of cells and $S$ the concentration of the chemical. The velocity space $V$ can be taken to be the unit ball but it is natural to assume that the velocity of the cells is constant and take $V$ to be the unit sphere [13].

Velocity fields on the sphere also arise in mathematical Physics in the phenomenon of radiative transfer which describes the scattering of photons in a hot medium $[1,2,21,30]$.

The results of this paper can be used to prove compactness of averages and hence global existence of weak solutions for the resonant Vlasov-wave system (3.3) below. Alternatively, the compactness of averages can be derived from the results of $[14,15]$.

$$
\begin{gathered}
\partial_{t} f+\frac{v}{|v|} \cdot \nabla_{x} f-\nabla_{x} u \cdot \nabla_{v} f=0, \quad\left(x, v \in \mathbb{R}^{3} t \in \mathbb{R}\right), \\
\partial_{t}^{2} u-\Delta u=-\mu, \quad \mu:=\int_{\mathbb{R}^{3}} f d v, \\
f(0, x, v)=f_{0}(x, v), \quad u(0, x)=u_{0}(x), \quad \partial_{t} u(0, x)=u_{1}(x) .
\end{gathered}
$$

\section{Acknowledgements}

The first author would like to thank the Laboratoire J.-L. Lions of Université P. et M. Curie (Paris 6) and the École Normale Supérieure for their hospitality and financial support during his sabbatical leave in the spring semester of 2008 when part of the work in this paper was done. The second author is supported by the China-France-Russia mathematics collaboration grant No. 340003275100 from Sun Yat-Sen University and he would like to thank Prof. Shangbin Cui, Vincent Calvez and Thibaut Allemand for several helpful discussions.

\section{References}

[1] C. Bardos, F. Golse and B. Perthame: The Rosseland approximation for the radiative transfer equations. Comm. Pure Appl. Math. 40 (1987), no. 6, 691-721.

[2] C. Bardos, F. Golse, B. Perthame and R. Sentis: The nonaccretive radiative transfer equations: existence of solutions and Rosseland approximation. J. Funct. Anal. 77 (1988), no. 2, 434-460.

[3] F. Bouchut: Introduction to the mathematical theory of kinetic equations, in F. Bouchut, F. Golse and M. Pulvirenti, Kinetic Equations and Asymptotic Theory, Series in Applied Mathematics, 4. Gauthier-Villars, Paris, 2000. x+162 pp.

[4] F. Bouchut and L. Desvillettes: Averaging Lemmas without time Fourier Transform and applications to discretized kinetic equations, Proc. Roy. Soc. Edinburgh, Sect. A 129, no. 1 (1999), 19-36.

[5] F. Bouchut, F. Golse and C. Pallard: Nonresonant smoothing for coupled wave+transport equations and the Vlasov-Maxwell system. Rev. Mat. Iberoamericana, 20 (2004), pp. 365-892. 
[6] N. Bournaveas and B. Perthame: Averages over spheres for kinetic transport equations: hyperbolic Sobolev spaces and Strichartz inequalities. J. Math. Pures Appl., 80 (2001), pp. 517-534.

[7] N. Bournaveas and S. Gutiérrez, On the regularity of averages over spheres for kinetic transport equations in hyperbolic Sobolev spaces. Rev. Mat. Iberoamericana 23 (2007), pp. 481-512.

[8] N. Bournaveas and S. Gutiérrez, Averages over spheres for kinetic transport equations with velocity derivatives in the right-hand side, SIAM J. Math. Anal. 40 (2008), no. $2,653-674$.

[9] N. Bournaveas, V. Calvez, S. Gutiérrez and B. Perthame: Global existence for a kinetic model of chemotaxis via dispersion and Strichartz estimates. Comm. Partial Differential Equations 33 (2008), no. 1-3, 79-95.

[10] F. A. C. C. Chalub, P. Markowich, B. Perthame and C. Schmeiser: Kinetic models for chemotaxis and their drift-diffusion limits. Monatsh. Math. 142 (2004), no. 1-2, $123-141$

[11] R. DeVore and G. Petrova: The averaging lemma. J. Amer. Math. Soc. 14 (2001), no. $2,279-296$

[12] R. J. DiPerna, P. L. Lions and Y. Meyer: $L^{p}$ regularity of velocity averages, Ann. Inst. H. Poincaré, Ann. Non Linéaire, Vol. 8 (1991), 271-287.

[13] Y. Dolak and C. Schmeiser: Kinetic models for chemotaxis: Hydrodynamic limits and spatio-temporal mechanisms. J. Math. Biol. 51 (2005), no. 6, 595-615.

[14] P. Gérard: Regularity of means of solutions of partial differential equations, Journées Équations aux Dérivées Partielles, 1987, p. 1-8.

[15] P. Gérard: Microlocal defect measures, Comm. Partial Diff. Eq., Vol. 16 (1991), 1761-1794.

[16] P. Gérard and F. Golse: Averaging regularity results for PDEs under transversality assumptions. Comm. Pure Appl. Math. 45 (1992), no. 1, 1-26.

[17] F. Golse, P. -L. Lions, B. Perthame and R. Sentis: Regularity of the moments of the solution of a transport equation, J. Funct. Anal., 76 (1988), pp. 110-125.

[18] F. Golse, B. Perthame and R. Sentis: Un résultat de compacité pour les équations de transport et application au calcul de la limite de la valeur propre principale d'un opérateur de transport, C. R. Acad. Sci. Paris Sér. I Math. 301 (1985), no. 7, 341344.

[19] F. Golse and L. Saint-Raymond: The Navier-Stokes limit of the Boltzmann equation for bounded collision kernels. Invent. Math. 155 (2004), no. 1, 81-161.

[20] F. Golse and L. Saint-Raymond: Velocity averaging in $L^{1}$ for the transport equation. C. R. Math. Acad. Sci. Paris 334 (2002), no. 7, 557-562.

[21] F. Golse and B. Perthame: Generalized solutions of the radiative transfer equations in a singular case. Comm. Math. Phys. 106 (1986), no. 2, 211-239.

[22] H. J. Hwang, K. Kang and A. Stevens: Global solutions of nonlinear transport equations for chemosensitive movement. SIAM J. Math. Anal. 36 (2005), no. 4, 1177-1199

[23] P.-E. Jabin and L. Vega: A real space method for averaging lemmas. J. Math. Pures Appl. (9) 83 (2004), no. 11, 1309-1351. 
[24] P.-E. Jabin and L. Vega: Averaging lemmas and the X-ray transform. C. R. Math. Acad. Sci. Paris 337 (2003), no. 8, 505-510.

[25] P.-E. Jabin and B. Perthame: Regularity in kinetic formulations via averaging lemmas. ESAIM Control Optim. Calc. Var. 8 (2002), 761-774

[26] P. L. Lions and B. Perthame: Lemmes de moments, de moyenne et de dispersion. C. R. Acad. Sci. Paris Sér. I 314, no. 11 (1992), 801-806.

[27] P. L. Lions: Régularité optimale des moyennes en vitesses, C.R. Acad. Sc., Séries I, 320 (1995), 911-915.

[28] B. Perthame: Mathematical tools for kinetic equations. Bull. Amer. Math. Soc. (N.S.) 41 (2004), no. 2, 205-244

[29] B. Perthame and P. E. Souganidis: A limiting case for velocity averaging, Ann. Scient. Ecole Normale Supérieure, 4ème série, 31, (1998), 591-598

[30] B. Perthame and J. L. Vázquez: Bounded speed of propagation for solutions to radiative transfer equations. Comm. Math. Phys. 130 (1990), no. 3, 457-469.

Nikolaos Bournaveas

University of Edinburgh

School of Mathematics

JCMB

King's Buildings

Edinburgh EH9 3JZ

United Kingdom

e-mail: N.Bournaveas@ed.ac.uk

Hua Wang

Sun Yat-Sen University

Department of Mathematics

Guangzhou, Guangdong 510275

P. R. China

Current address:

École Normale Supérieure

Département de Mathématiques et Applications

45 rue d'Ulm

F-75230 Paris Cedex 05

France

e-mail: wanghua_math@126.com

Received: 4 October 2008.

Accepted: 10 December 2008. 